detect the residual activities of platelet induced by thrombin AA or $\mathrm{ADP}$. And then the inhibition ratios of platelet after therapy were calculated and the characteristics of their distribution were analysed.

Results 1) The maximal potential activities of platelet are not homogeneous. Among the patients enrolled in this study, $7.1 \%$ is in very low activity while $14.3 \%$ is in very high. 2) The inhibition on aggregation of platelet also differs in $\mathrm{ADP}$ pathway and $\mathrm{AA}$ pathway. The frequency fractional of aspirin is more lower when the inhibition rate is under $30 \%$ and more higher when it is between $70 \%-79.9 \%(p<0.05) .3)$ The actual frequency fractional variation of each intensity of inhibition also differs significantly $(p=0.0026)$. 4) Even in the same patient the inhibition of platelet aggregation of the two pathways, AA and ADP, is not synchronous. 5) Only $1.6 \%$ of the patients experienced resistance in both aspirin and clopidogrel pathway, and $3.8 \%$ of them are over-sensitive in both.

Conclusion We should assess basic activity of platelet and the reacts to remedies on every patient individually because they differ significantly in each case. Even in same patient, the change of inhibition on aggregation of platelet by aspirin or clopidogrel is not synchronous. So, we should assess the effects of aspirin and clopidogrel respectively in each patient. In Chinese patients with ACS, the inhibition intensity of platelet by aspirin with regular dosage is higher than that of clopidogrel. Only $1.6 \%$ patients with both aspirin and clopidogrel resistance, who are at high risk of thrombosis, while $3.8 \%$ patients over-sensitive to both aspirin and clopidogrel, who are at high risk of haemorrhage. All of these mean that the individual assessment on activity of platelet and reaction of antiplatelet therapy should been done in order to adjust medicine and dosages.

\section{e0459 PRIMARY EVALUATION TO SAFETY AND VALUE OF IABP IN PATIENTS WITH ACUTE CORONARY SYNDROME COMPLICATED WITH PUMP FAILURE}

doi:10.1136/hrt.2010.208967.459

Wu Keng, He Songjian. The Affiliated Hospital of Guangdong Mediacal Collegue/ Department of Cardiology

Objective To evaluate the therapeutic effect and safety, and the result of coronary angiography (CAG) and serum brain natriuretic peptide (BNP) level in patients with acute coronary syndrome complicated with pump failure treated with intra aortic balloon pump (IABP).

Methods Between January 2008 and May 2009, 121 patients with acute coronary syndrome (ACS) and pump failure were divided into IABP group (61 cases) and the control group (60cases), respectively. There were 76 male patients and 45 female patients, while 77 were ST-elevation myocardial infarction (STEMI), 35 were Non STelevation myocardial infarction (NSTEMI) and 9 were Unstable angina pectoris (UA).40 patients fell into Killip rank-II, 45 patients fell into [i]Killip rank-III, while 36 patients fell into Killip rank-IV. All of them were suitable for IABP treatment and there was no contraindication. They all received basical treatment, including antianginal therapy, anti-platelet, cholesterol lowing, eatl. All patients underwent $\mathrm{CAG}$ and percutaneous coronary intervention (PCI). The IABP group were treated with IABP while the control group were not. Evaluations 1. Clinical effect (including the effect of angina and pump failure), timing in hospital, death rate in $30 \mathrm{~d} 2$. The CAG result after PCI. 3. The level of EF measured by cadioultrasound. 4. The level of BNP were measured.

Results After treatment, the IABP group were higher significantly than the control group in clinical effect, including the effect of angina and pump failure $[94.7 \%$ vs $71.6 \%$ ( 0.05 )] IABP group was lower than the control group on the BNP after treatment, there were significantly difference in two groups $(4893 \pm 966 \rightarrow 316 \pm 91$ vs
$4687 \pm 912 \rightarrow 511 \pm 120(\mathrm{ng} / \mathrm{l}) \quad(\mathrm{p}<0.01)$. As for the complication, there were few patients with bleeding $(3.2 \%(2 / 61))$, haematoma $(3.2 \%(2 / 61))$, acute earterial embolism $(1.6 \%(1 / 61))$, fever $(1.6 \%(1)$ $61)$ ), platelet decreasing $(4.8 \%(3 / 61))$. Although there were more complications in IABP group, after treatment the symptom got advanced and there were no severe complication.

Conclusion IABP can significantly advance the haemodynamic indexes and serum BNP level of the patients of ACS with pump failure, can ease the pain of Angina, improve the tolerance and successful rate of the PCI treatment, and decrease death rate. And there is no severe complication, and it is a kind of safe treatment.

\section{e0460 EFFECT OF SHENGMAI INJECTION (SMI) ON BLOOD VASCULAR ENDODERMIS FUNCTION AND CARDIAC FUNCTION IN CORONARY ARTERY DISEASE WITH TYPE 2 DIABETES PATIENTS}

doi:10.1136/hrt.2010.208967.460

Zhang Yachen, Chen Man-Tian, Wang Fei, Rong Yezhi, Lu Baojing. Division of Cardiology, Xinhua Hospital, School of Medicine, Shanghai Jiaotong University

Objective To investigate the drug effects of Shengmai Injection (SMI) on blood vascular endodermis function and cardiac function in coronary artery disease (CAD) with type 2 diabetes mellitus (2DM) patients.

Methods A total of 120 patients with type 2 diabetes mellitus and CAD diagnosed by coronary angiography (CAG) were randomised into two groups, the control group, treated with conventional therapy and the SMI group, treated with SMI. The patients ' nitrite oxide (NO), Endothelin-1 (ET-1), AngII in venous blood were noted, and the blood vascular endodermis function, cardiac function were measured before treatment and after 3 weeks treatment, so as to observe and compare their changes in the two groups.

Results After 3 weeks of treatment, the serum content of NO was significantly higher, the serum content of ET-1 and AngII were significantly lower in the SMI group than that in the control group $(p<0.01)$; The patients' blood vascular endodermis function and cardiac function were improved in SMI group than that in the control group $(\mathrm{p}<0.01)$.

Conclusion The SMI could not only improve the blood vascular endodermis function but also improve the cardiac function in coronary artery disease with type 2 diabetes mellitus patients.

\section{E0461 THE APPLICATION PROSPECTS OF ORAL FXA INHIBITORS IN ACUTE CORONARY SYNDROME}

doi:10.1136/hrt.2010.208967.461

Li Yuanmin, Li Yuanmin. Shanghai 10th People's Hospital

Acute coronary syndrome (ACS) patients would still have twice heart attack, even after they had received the standard regimen, such as vascular reconstruction, oral anti-platelet therapy, and other evidence based medical therapy. Therefore, clinical have been in urgent need of a new and effective anticoagulant. OASISresearch have shown that the factor Xa play a decisive role in the process of coagulation. For ACS patients' anticoagulant therapy, compared with normal or low molecular weight heparin, the FXa indirect inhibitor Fondaparinux sodium can not only be effective in reducing cardiovascular events, but also greatly reduce the risk of bleeding. It is especially for the patients with higher risk of bleeding and regardless the patient's age, gender, renal function and risk stratification. Based on these findings, a variety of clinical trials 